\title{
Recent results on Electroweak measurements from ATLAS
}

\author{
Nektarios Chr. Benekos ${ }^{1, a}$, on behalf of the ATLAS Collaboration \\ ${ }^{1}$ Department of Physics, National Technical University of Athens, Hellas
}

\begin{abstract}
ATLAS measurements of multiboson production processes involving combinations of $\mathrm{W}, \mathrm{Z}$ and isolated photons are summarized. Measurements using data at 7 $\mathrm{TeV}$ and at $8 \mathrm{TeV}$ are presented. The measurements are performed using leptonic decay modes, including the invisible decay $Z \rightarrow v \bar{v}$, as well as semileptonic channels. Measurements of single and diboson production in association with two forward jets is sensitive to electroweak vector boson fusion and scattering processes. An observation of the electroweak production of the $\mathrm{Z}$ boson and an evidence of same sign WW production are reported.
\end{abstract}

\section{Introduction}

Precision measurements of electroweak (EW) processes in pp collisions provide excellent precision tests of the EW sector of the Standard Model (SM), and help to constrain the parton density functions (PDF) of the proton. Measurements of vector boson pair production are essential not only in their own right, but also for measurements of Higgs and relevant new physics searches, since they are the main backgrounds for those analyses. In addition, a precise measurement of the vertices, through the analysis of diboson production, is essential to test the high energy behavior of EW interactions and to probe possible new physics in the bosonic sector. Any deviation from SM gauge constraints can cause a significan enhancement in the production cross section at high diboson invariant mass due to anomalous triple gauge boson couplings (aTGC). The expected triple (TGC) and quartic (QGC) gauge boson couplings can be tested at the Large Hadron Collider (LHC) through studies of multiboson $(W, Z, \gamma)$ production or through studies of vector boson fusion (VBF) and vector boson scattering (VBS) processes. Studies of VBS processes are also of interest as they can potentially test whether the single Higgs scalar boson of the SM is indeed solely responsible for unitarising the amplitude for longitudinal $\mathrm{W}$ boson scattering, $W_{L} W_{L} \rightarrow W_{L} W_{L}$. This note presents measurements of the diboson and EW production cross sections in proton-proton collisions at centre-of-mass energies of $\sqrt{s}=7 \mathrm{TeV}$ and $8 \mathrm{TeV}$ using data recorded by the ATLAS detector [1] at the LHC [2] in 2011 and 2012. The datasets analyzed correspond to an integrated luminosity of $4.7 \mathrm{fb}^{-1}$ and $20.3 \mathrm{fb}^{-1}$ respectively. The cross sections of the studied processes are calculated at Next to Leading Order (NLO) in QCD using MCFM [3] with PDF set CT10 [4]. In addition, the prospects for studies of VBF and VBS processes via measurements of electroweak Zjj and $W^{ \pm} W^{ \pm} j j$ production are also discussed.

\footnotetext{
a e-mail: nectarios.benekos@cern.ch
} 


\section{Cross section measurement and common topics}

The diboson fina state measurements depends on the quality of the reconstructed objects as well as on the sub-detector resolutions. All the presented measurements have leptons in their fina states, plus other objects as photons, jets or missing transverse energy $\left(E_{\mathrm{T}}^{\mathrm{miss}}\right)$. As a consequence, the boson + jets and the $t \bar{t}$ event categories are always present among the background processes: a jet can fake an electron or a gamma, or may be selected in hadronic fina states, while the top contribution is important because it provides real W-bosons and jets. Other background contributions come from multijet events and pile-up. The selections discussed in the this review have been introduced to decrease the contributions of the backgrounds in the various analyses without deteriorating the efficien y of the signal selection. In the following, six diffent diboson cross section measurements are briefl described, with a focus on the differences of the fina states, backgrounds and cut applied. The references to the articles explaining the specifi measurements are reported.

\subsection{Physics Objects definitio}

In all the presented analyses, the $\mathrm{W}$ and $\mathrm{Z}$ bosons are reconstructed from their decay products, trigering the events on the presence of a lepton above a given $p_{\mathrm{T}}$ threshold. Selections are usually applied on lepton and jet reconstruction quality, multiplicity, angular requirements, as well as on the $E_{\mathrm{T}}^{\mathrm{miss}}$ and event overall quality. The corresponding physical objects are define in ATLAS as follows:

- electrons: a candidate electron is obtained from an energy cluster in the electromagnetic (EM) calorimeter, providing the particle energy, associated with a reconstructed track in the Inner Detector (ID), which gives information on the direction; the electrons are usually requested to be isolated both using tracks and calorimetric informations;

- muons: muons are reconstructed matching the tracks from the Muon Spectrometer to those from the Inner Detector, and requiring isolations from other tracks and energy deposits in calorimeters;

- jets: identifie as energy deposits in both electromagnetic and hadronic calorimeters; the energetic clusters are processed by the antiKt jet reconstruction algorithm [5], with cone sizes $\Delta R=0.4$ or $\Delta R=0.6$. The antiKt algorithm is used because it is stable, fast, infrared and collinear-safe. A calibration factor dependent on the cluster $p_{\mathrm{T}}$ and $\eta$ is applied to correct for detector and physics effects [6]. The jet energy and direction is obtained from the ensemble of calorimetric clusters of energy associated with the jet;

- missing transverse energy: the $E_{\mathrm{T}}^{\text {miss }}$ is a complex object which describes the unbalanced transverse momentum in each event; it represents a vector lying in the transverse plan and it is sensitive to the presence of neutrinos; the $E_{\mathrm{T}}^{\text {miss }}$ depends also on the pile-up conditions and on the calibrations and resolutions of the physics objects described above.

\section{Diboson production}

\section{1 $W \gamma$ and $Z \gamma$ production}

In this process a massive boson is produced in association with a high energy photon [7]. The diboson candidate events are selected requiring a lepton, $E_{\mathrm{T}}^{\mathrm{miss}}$ and a photon, or two opposite fl vor leptons and a photon, or large $E_{\mathrm{T}}^{\mathrm{miss}}$ and a photon. These fina states include also the photon bremsstrahlung from the leptons for the $W / Z$ decays. Events with massive bosons are identifie with cuts on the transverse mass (for $\mathrm{W}$ ) or on the invariant mass $(\mathrm{Z})$ of the leptonic fina states, and an isolated photon is requested, with a large angular separation from the leptons. To suppress the contribution of 


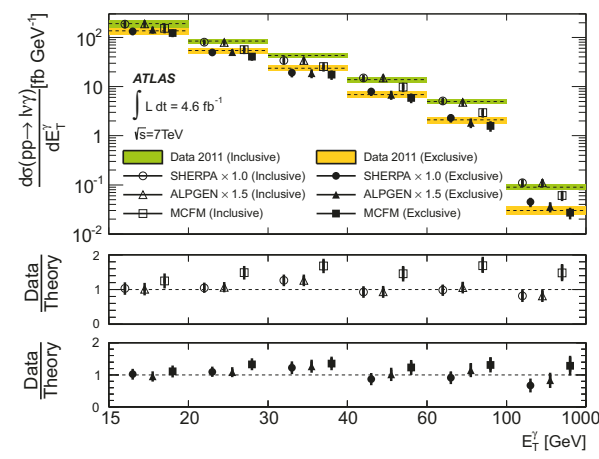

(a)

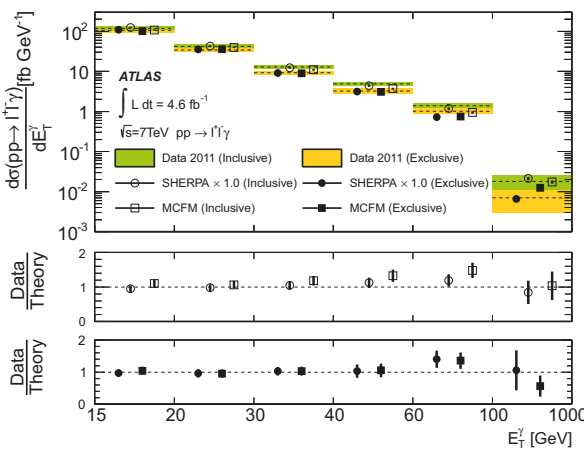

(b)

Figure 1: Measured $E_{\mathrm{T}}^{\gamma}$ differential cross sections of the (a) $p p \rightarrow W \gamma \rightarrow \ell v \gamma$ and (b) $p p \rightarrow Z \gamma \rightarrow$ $\ell^{+} \ell^{-} \gamma(\mathrm{b})$ processes, using combined electron and muon measurements in the inclusive $\left(N_{\text {jet }} \geq 0\right)$ and exclusive $\left(N_{\text {jet }}=0\right.$ with $\left.p_{\mathrm{T}}>30 \mathrm{GeV}\right)$ extended fiducia regions in the ATLAS experiment. The resulting measured and expected inclusive and exclusive cross sections for $W \gamma$ and $Z \gamma$ production processes are compatible within uncertainties. The lower plots show the ratio of the data to the predictions by different generators. The empty circles, triangles and boxes (top plots of the ratio panels) refer to the inclusive measurements, the full symbols refer to the exclusive selection (bottom plots of the ratio panels).

Final State Radiation (FSR), the photon was required to be separated with lepton by $\Delta R>0.7$. Main backgrounds $(W / Z / \gamma+j e t s)$ were estimated with two-dimensional sideband method. Cross section measurements are performed in the inclusive and exclusive fina state, depending on the number of jets produced. The candidate events for $W \gamma$ and $Z \gamma$ production are selected from the production processes $p p \rightarrow \ell v \gamma+X, p p \rightarrow \ell^{+} \ell^{-} \gamma+X$ and $p p \rightarrow v v \gamma+X$ [7]. Figure 1 shows the signal differential cross section, for both the inclusive and exclusive measurements at particle level (where the detector effects have been unfolded) as a function of the photon transverse momentum $\left(E_{\mathrm{T}}^{\gamma}\right)$, for data and Monte Carlo (MC). In general, quite a good agreement is found between data and both the parton-shower (SHERPA [8] and ALPGEN [9]) and the NLO genetator (MCFM [3]) calculations, the only disagreement being at large $E_{\mathrm{T}}$ for MCFM. This is due to the fact that the MCFM calculation used are up to 1 jet, and generally the high $p_{\mathrm{T}}$ region is not very well described. The measurements do not highlight any deviations from the SM predictions for the $V \gamma$ production cross section.

\subsection{WW production}

The $W^{+} W^{-} \rightarrow \ell^{+} \ell^{-}$signal is measured in fina states with two opposite-sign isolated leptons and large missing transverse energy [10]. Two high- $p_{\mathrm{T}}$ isolated leptons $(1=\mathrm{e}$ or $\mu)$ were required with opposite charge. To reject $t \bar{t}$ and $\mathrm{W}+$ jets background, events were vetoed if any jet had $p_{\mathrm{T}}>25 \mathrm{GeV}$. This caused the dominant systematic uncertainty in the measurement. Events with same fl vor leptons were required to be out of the $\mathrm{Z}$ mass window to reject $\mathrm{Z}+$ jets background. The measured total WW production cross section amounts to $\sigma_{\text {tot }}=71.4_{-1.2}^{+1.2}$ (stat. - $_{-4.4}^{+5.0}$ (syst. $)_{-2.1}^{+2.2}$ (lumi) pb for $\sqrt{s}=8 \mathrm{TeV}$, compared to a theory prediction $\sigma_{\text {tot }}^{\text {theory }}=58.7_{-2.7}^{+3.0} \mathrm{pb}$. Figure 2(b) shows the cross sections measured in the individual channels as well as the combined cross section and compares them to the SM 


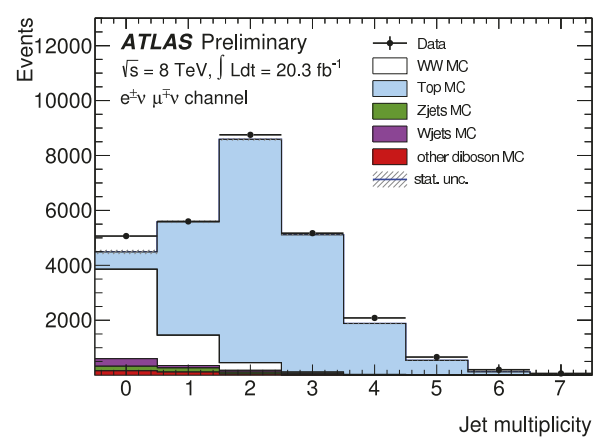

(a)

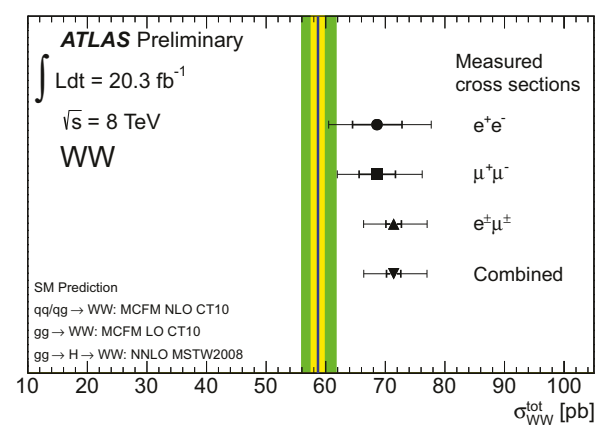

(b)

Figure 2: (a): Jet multiplicity distribution in $W^{+} W^{-}$events for the $e^{ \pm} \mu^{\mp}$ channel, without imposing the jet veto requirement (which selects only the firs bin) [10]. (b): Comparison between predicted $W^{+} W^{-}$production cross-section using CT10 PDF and the measured cross section in $e^{ \pm} \mu^{\mp}, e^{+} e^{-}$, $\mu^{+} \mu^{-}$and combined channels.he yellow and green shaded bands represent the PDF and total theoretical uncertainties respectively. The fille symbols show the measured total cross section with the statistical and total uncertainty [10].

prediction obtained using the PDF set CT10 [4]. The measured combined cross section differs from the SM prediction computed using CT10 PDF by $2.1 \sigma$ using the standard PDF and scale uncertainties. Various contributions to the total cross section like soft-WW $p_{\mathrm{T}}$ resummation $[11,12]$; have been neglected here and might cause an increase of a few percent to the predicted cross section.

\section{3 $\mathrm{ZZ}$ production}

The analyses of $Z$ boson pair production is performed in the $4 \ell$ and $2 \ell 2 v$ fina states $[13,14] . Z Z \rightarrow$ $\ell^{+} \ell^{-} \ell^{+} \ell^{-}$signature comprises four isolated leptons and the invariant mass of each reconstructed opposite-sign same-fl vor lepton pair forming a $Z$ is required to be within $66 \mathrm{GeV}<m_{\ell \ell}<116 \mathrm{GeV}$. For the $Z Z \rightarrow \ell^{+} \ell^{-} v v$ channel the signature is two opposite-sign same-fl vor high $p_{\mathrm{T}}$ isolated leptons within $76 \mathrm{GeV}<m_{\ell \ell}<106 \mathrm{GeV}$. The total measured $\mathrm{ZZ}$ production cross sections are $\sigma_{\text {tot }}=6.7 \pm$ 0.7 (stat.) ${ }_{-0.3}^{+0.4}$ (syst.) \pm 0.3 (lumi) pb for $\sqrt{s}=7 \mathrm{TeV}$ and $\sigma_{\text {tot }}=7.1_{-0.4}^{+0.5}$ (stat.) \pm 0.3 (syst.) \pm 0.2 (lumi) pb for $\sqrt{s}=8 \mathrm{TeV}$, and the theory predictions are $\sigma_{\text {tot }}^{\text {theory }}=5.89_{-0.18}^{+0.22} \mathrm{pb}$ and $\sigma_{\text {tot }}^{\text {theory }}=7.2_{-0.2}^{+0.3} \mathrm{pb}$ for $\sqrt{s}=7 \mathrm{TeV}$ and $\sqrt{s}=8 \mathrm{TeV}$ respectively. Figure 4(a) illustrates the comparison of experimental measurements and theoretical predictions of the total $\mathrm{ZZ}$ production cross section as a function of the centre-of-mass energy.

\subsection{WZ production}

The analysis of WZ production is performed with $\left(\ell^{+} \ell^{-}\right) \ell^{\prime \pm} v$ fina states $[15,16]$. The signature of these topologies is two opposite-sign same-fl vor isolated leptons forming the $\mathrm{Z}$ boson within a tight invariant mass window around the $\mathrm{Z}$ pole of $\left|m_{Z}-m_{\mathrm{PDG}}\right|<10 \mathrm{GeV}$ which suppresses the background significantl. The residual third isolated lepton and the $E_{\mathrm{T}}^{\mathrm{miss}}$ are forming the $\mathrm{W}$-boson. Main sources of background are the $\mathrm{Z}+$ jets and $t \bar{t}$ events where the two lep- 


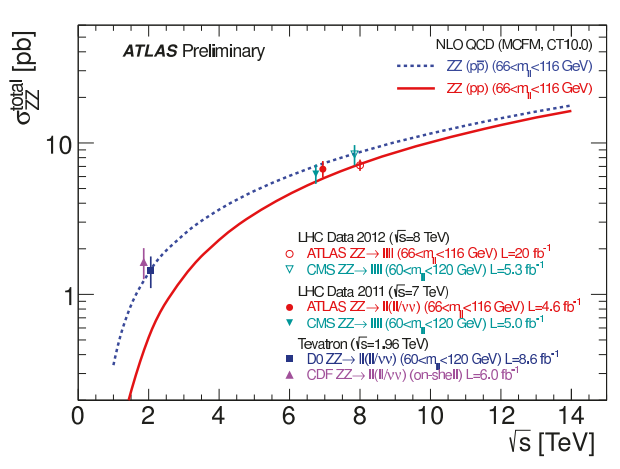

(a)

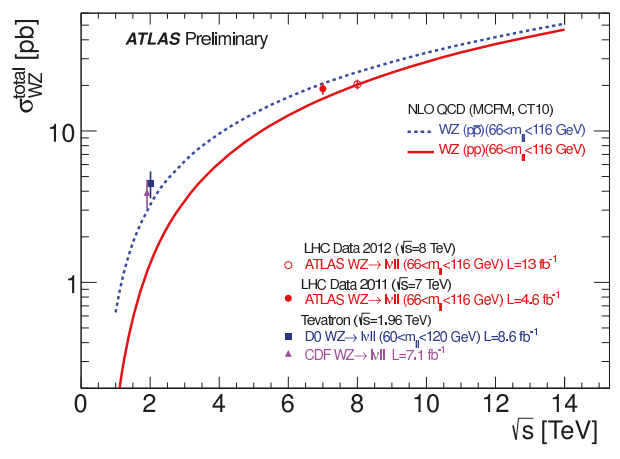

(b)

Figure 3: (a): Comparison of experimental measurements and theoretical predictions of the total ZZ (a) and WZ (b) production cross section as a function of the centre-of-mass energy $\sqrt{s}$. The solid red line shows the theoretical prediction for the WZ production cross section in pp collisions, calculated using the natural width of the $\mathrm{Z}$ boson in the mass range 66 to $116 \mathrm{GeV}$.

tons from the vector boson decays are accompanied by a jet, which is misidentifie as a lepton. These backgrounds were estimated from data-driven techniques. The total WZ production cross section measurements are $\sigma_{\text {tot }}=19.0_{-1.3}^{+1.4}$ (stat.) \pm 0.9 (syst.) \pm 0.4 (lumi) pb for $\sqrt{s}=7 \mathrm{TeV}$ and $\sigma_{\text {tot }}=20.3_{-0.7}^{+0.8}$ (stat. $)_{-1.1}^{+1.2}$ (stat. $)_{-0.6}^{+0.7}$ (lumi) pb for $\sqrt{s}=8 \mathrm{TeV}$ while the SM expectation is $\sigma_{\text {tot }}^{\text {theory }}=$ $17.6_{-1.0}^{+0.22} \mathrm{pb}$ and $\sigma_{\text {tot }}^{\text {theory }}=20.3 \pm 0.8 \mathrm{pb}$ for $\sqrt{s}=7 \mathrm{TeV}$ and $8 \mathrm{TeV}$, respectively. In Figure 4(b) experimental measurements and theoretical predictions of the total WZ production cross section as a function of the centre-of-mass energy $\sqrt{s}$ are compared. The total cross section is calculated using the natural width of the $\mathrm{Z}$ boson in the mass range 66 to $116 \mathrm{GeV}$.

\subsection{WW and WZ production in the single lepton fina state}

WW and WZ production where one $\mathrm{W}$ or the $\mathrm{Z}$ boson decays into jets are also established for $\sqrt{s}=7$ $\mathrm{TeV}$ [17]. Candidate events have one high $p_{\mathrm{T}}$ isolated lepton, $E_{\mathrm{T}}^{\text {miss }}>30 \mathrm{GeV}$ and exactly two jets with $p_{\mathrm{T}}>25 / 30 \mathrm{GeV}$. The semileptonic diboson fina state tests the ability of the experiment to reconstruct dijet invariant masses in an environment in which the cross section for the jet production is very high. The most important backgrounds for this channel are the $\mathrm{W}+$ jets and the $\mathrm{Z}+$ jets processes. Final states with a top quark contribute to the background with a real W-boson, but they can be reduced with a jet multiplicity cut. The candidate jets are ordered in $p_{\mathrm{T}}$ and only the leading and the subleading jets are considered to construct the boson mass. Figure 4(a) shows the dijet invariant mass distributions of reconstructed $W / Z \rightarrow j j$ candidates and the background subtracted dijet invariant mass distribution of reconstructed $W / Z \rightarrow j j$ candidates candidates. The $\mathrm{W} / \mathrm{Z}$ resonance has been observed with 3.3 $\sigma$, and the mesured cross section is $\sigma_{\text {tot }}=72 \pm 9$ (stat.) \pm 15 (syst.) \pm 13 (MC stat.) pb compared to the theory prediction of $\sigma_{\text {tot }}^{\text {theory }}=63.4 \pm 2.6 \mathrm{pb}$ 


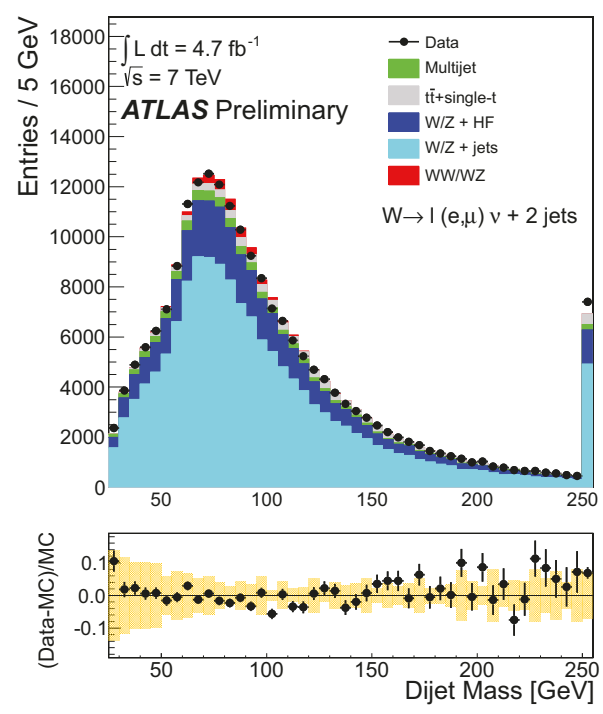

(a)

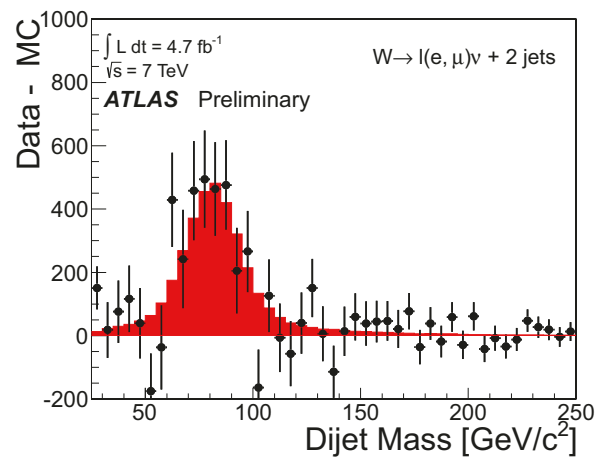

(b)

Figure 4: (a): dijet invariant mass distribution of reconstructed $W / Z \rightarrow j j$ jj candidates. The rightmost bin includes the overfl $\mathrm{w}$. The lower panel displays the fractional difference between the data and the MC expectation. The yellow band displays the systematic uncertainty due to the JES uncertainty only. (b): Background subtracted dijet invariant mass distribution of reconstructed $W / Z \rightarrow j j$ candidates. The errors bars represents statistical uncertainty of data and MC.

\section{Electroweak production of $Z$ bosons in association with two jets}

The dominant production process for a $\mathrm{Z}$ boson at the LHC is via the Drell Yan process. Additional jets arise from strong interactions like gluon radiation or gluon splitting. A $Z$ boson in association with two jets can also be produced in pure electroweak processes via t-channel exchanges of a vector bosons. As these processes contain no strong interactions they are much rarer than the $\mathrm{Z}$ boson + 2 jets production including strong interactions. Examples of such processes are shown in Figure 5. The most interesting pure electroweak process is the Feynman diagram shown on Figure 5(a), where the $\mathrm{Z}$ boson is produced via VBF of two $\mathrm{W}$ bosons. The two jets in the fina state, also called the "tagging" jets, occur directly from the incoming quarks and show a characteristic kinematic. They are often scattered in the very forward and backward region of the detector. Since there is no color exchange between the incoming quarks, no hadronic activity is expected in the central region of the detector. The large angle between the two jets leads to a large invariant mass of the dijets system, which allows to identify the VBF process. This process is similar to the Higgs production via VBF and can therefore be used to understand the kinematics of the "tagging" jets, which is needed to study the Higgs boson properties, especially in models where the Higgs boson does not couple to leptons and the production via VBF becomes the dominant process. Figure 5(c) shows the t-channel excange of the $\mathrm{W}$ boson, in this process the $\mathrm{Z}$ boson is radiated from one of the quark lines. The processes in Figure 5 have a large interference so they can not be calculated separately. The largest 


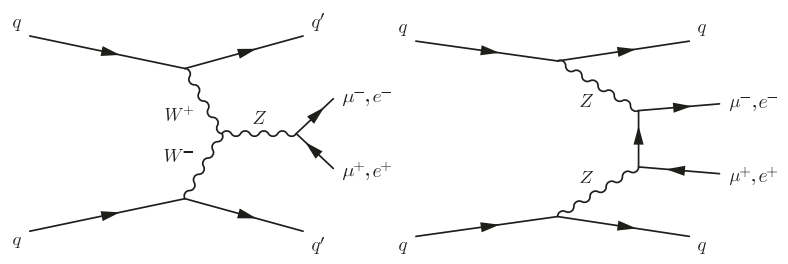

(a)

(b)

Figure 5: Feynman diagrams of pure electroweak $\mathrm{Z}+$ two jets production: (a): shows the VBF process, where the $\mathrm{Z}$ boson is produced via the fusion of two Vector Bosons. (b): shows the nonresonant production.

challenge for measuring the pure electroweak process is to separate it from the large background of $\mathrm{Z}$ boson with two quarks from strong interactions. In the following the different approaches for the cross section measurement from both experiments ATLAS and CMS and some highlights from studies on the hadronic interaction between the tagging jets are discussed. Besides the cross section measurement of $\mathrm{Z}+2$ jets in f ve fiducia regions with different sensitivity to the pure electroweak component, ATLAS provides also the unfolding of various VBF sensitive distributions and limits on anomalous triple gauge couplings. Figure 6(a) shows the results of the cross section measurement in the different kinematic regions. Apart from the baseline selection which requires only a nominal $\mathrm{Z}+2$ jets selection with two isolated leptons and two jets, the "high $p_{\mathrm{T}}$ " region cuts at $p_{\mathrm{T} \text { jet } 1 / \mathrm{jet} 2}>$ $85 / 75 \mathrm{GeV}$, while the "high mass" region cuts at $m_{\mathrm{jj}}>1 \mathrm{TeV}$. The last two region are optimised to enhance the fraction of the pure electroweak component ("search region") and to suppress it ("control region"), by suppressing or requiring a third jet between the tagging jets. In the control region the background modelling is studied and could be improved by a reweighting procedure, which is then also applied in the signal region. The cross section in all fiducia regions is extracted by a fi to the invariant mass distribution of the dijet system. Figure 6(a) shows that in all phase spaces the measured cross section in data is in good agreement with theory prediction from POWHEG and SHERPA. Figure 6(b) shows the unfolded invariant dijet mass in data in the search region compared to POWHEG and SHERPA. POWHEG describes the data better as it is accurate to Next-to-Leading-Order in QCD as SHERPA is only accurate in leading order. The measured fiducia cross sections in the search region $m_{\mathrm{jj}}>250 \mathrm{GeV}$ is $\sigma_{\mathrm{EW}}=54.7 \pm 4.6(\mathrm{stat})_{10.4}^{+9.8}$ (syst) \pm 1.5 (lumi)fb compared to the theory prediction of $\sigma_{\text {theory }}=46.1 \pm 0.2(\text { stat })_{0.2}^{+0.3}$ (scale) \pm 0.8 (PDF) \pm 0.5 (model) $)$ fb. Further details on this analysis can be found in [18].

\section{Vector boson scattering: $W^{ \pm} W^{ \pm} j j$ production}

The scattering of two massive vector bosons (VBS) is a key process to probe the nature of electroweak symmetry breaking. Even after the discovery of the SM-like Higgs boson on 2012 many physics scenarios predict enhancements in VBS either from additional resonances or if the observed Higgs boson only partially unitarizes the WW scattering amplitude. Examples of electroweak VBS contributions to the process $q q \rightarrow W W j j$, involving $W W V V(V=W, Z)$ QGCs or Higgs exchange, are shown in Figure 7. Similarly to the case of $Z j j$ production above, the VBS processes are accompanied by other electroweak contributions and are dominated by strong (QCD) contributions. Again, the VBS diagrams for $W W j j$ production tend to produce two high $p_{\mathrm{T}}$, forward jets, and the VBS contribution 


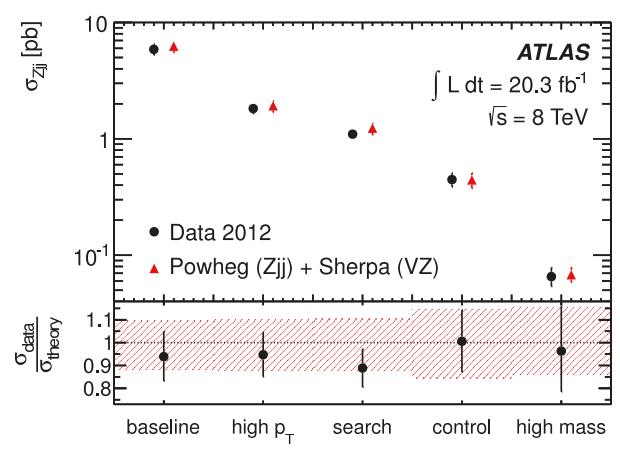

(a)

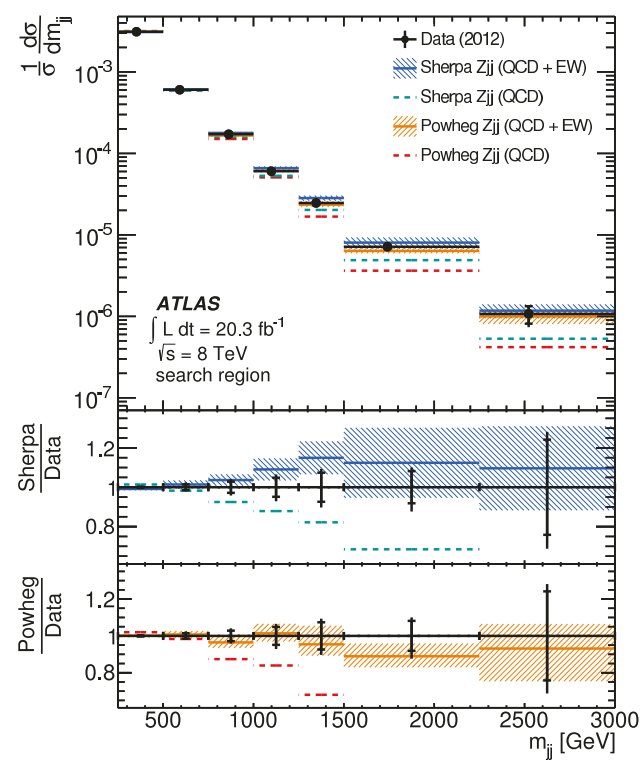

(b)

Figure 6: (a): Fiducial cross section measurements for inclusive Zjj production in the $\ell^{+} \ell^{-}$decay channel, compared to the POWHEG prediction for strong and electroweak Zjj production and the small contribution from diboson initiated Zjj production predicted by SHERPA [12]. (b): The dijet invariant mass distribution in the search region. The signal and (constrained) background templates are scaled to match the number of events obtained in the fit The lowest panel shows the ratio of constrained and unconstrained background templates to the data. The vertical error bars show the size of the total uncertainty on the measurement, with tick marks used to reflec the size of the statistical uncertainty only. Particle-level predictions from SHERPA and POWHEG are shown for combined strong and electroweak Zjj production ( labelled as QCD+EW) by hatched bands, denoting the model uncertainty, around the central prediction, which is shown as a solid line. The predictions from SHERPA and POWHEG for strong Zjj production (labelled QCD) are shown as dashed lines.

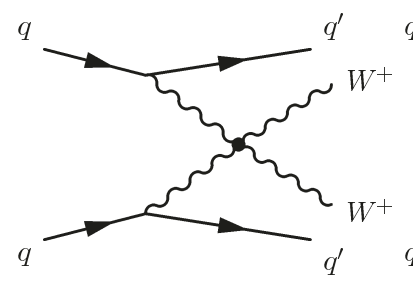

(a)

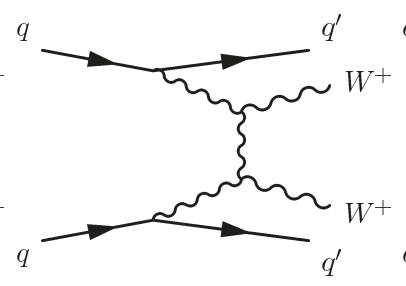

(b)

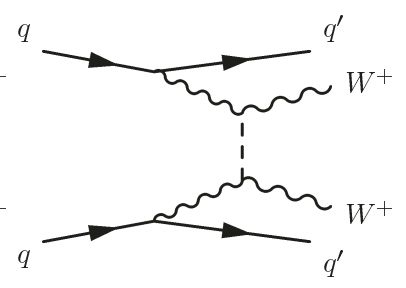

(c)

Figure 7: Examples of electroweak VBS Feynman diagrams contributing to $W^{ \pm} W^{ \pm} j j$ production. 


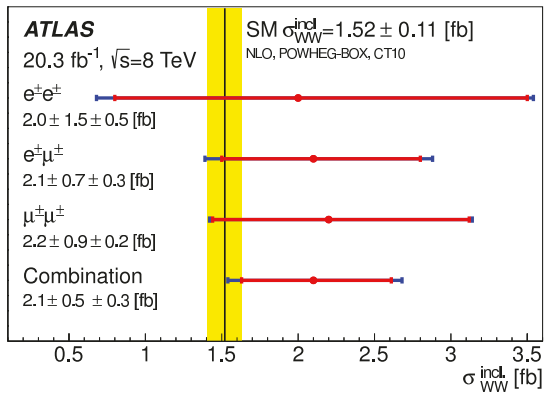

(a)

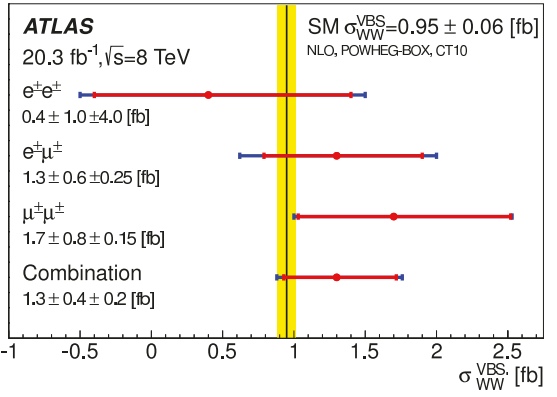

(b)

Figure 8: Results of the cross section measurements for VBS in the inclusive (a) and in the VBS enhanced (b) phase space for the different lepton channels as well as for the combination. The inner red error band represents the statistical error, and the blue band represents the total error on each measurement. In all channels and in both phase spaces the results agree within the uncertainties with the SM.

can be enhanced by requiring large invariant mass, $m_{\mathrm{jj}}$, or large rapidity separation, $\left|\Delta y_{\mathrm{jj}}\right|$, of the two forward jets. Unlike Zjj production, and since at Leading Order (LO) the two W bosons produced in $g g \rightarrow W W j j$ processes must have opposite electric charge, the QCD contribution to $\mathrm{WWjj}$ production can be reduced by a large factor by requiring that the two W-bosons have the same charge. Like in the VBF process, same-sign $W^{ \pm} W^{ \pm} j j$ events are selected in the $8 \mathrm{TeV}$ data [19] and the event topology is dominated by the "tagging" jets, the high momentum forward jets, which occur directly from the incoming quarks. Also here, a high invariant mass of the dijet system is characteristic for VBS events. In addition to the two jets, two high energy leptons from the $\mathrm{W}$ decays and large $E_{\mathrm{T}}^{\text {miss }}$ are expected. To suppress background from Drell Yan and top-pair production the signal selection requires leptons with the same charge. The cross section for the electroweak process is measured in two different kinematic regions, which require both $m_{\mathrm{jj}}>500 \mathrm{GeV}$. Once in the "VBS" region with an additional cut on $\Delta R(j j)>2.4$, where the VBS component is enhanced and in a more inclusive region without the $\Delta R(j j)$ cut. The measurement of the cross section is, in both regions, performed in three different channels based on the lepton fl vor: $e e, \mu \mu, e \mu$. The results are shown in Figure 8(a), for the inclusive and on Figure 8(b) for the VBS region. The expected production cross sections for the $p p \rightarrow W^{ \pm} W^{ \pm} j j$ process in the two fiducia regions ("fiducia cross sections") are calculated using POWHEG [20], [21] with CT10 parton distribution functions (PDFs) [4], interfaced with Pythia8 [22], [23] for parton showering, hadronization, and underlying event modeling. Contribution from non-resonant production of the same leptonic fina state is also considered, but is strongly suppressed [21]. The measured cross sections in all lepton channels separately, as well as the combination, agree well with the SM prediction.

\section{Summary and Conclusion}

The results for six different diboson production and decay channels obtained with the ATLAS detector at the LHC have been discussed, highlighting the excellent performance of both the accelerator and 


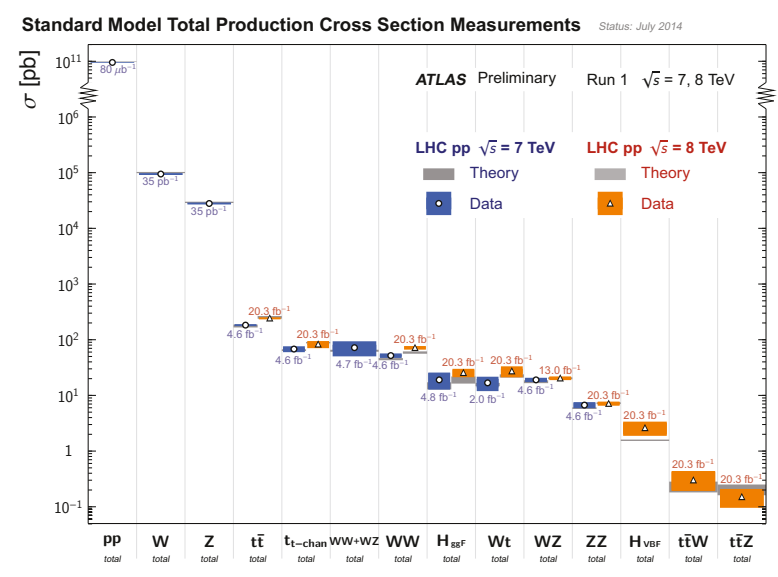

Figure 9: Summary plot of the SM measurements in the Electroweak sector with the ATLAS detector using both 2011 and 2012 data [18]. The diboson tipical cross sections are about 3-4 order of magnitude smaller than the diboson background processes.

the experiment, all the analyses being limited by systematic uncertainties. Figure 9 reports some of the diboson cross section measurements and offers a comparison with other processes which act as backgrounds to the diboson fina states, spanning a range of more than three orders of magnitude. The measurements shows no significan deviations from the SM predictions, and data and MC agree quite well with NLO calculations. Measurements of electroweak diboson production with the ATLAS experiment continue to improve. Total and fiducia production cross section measurements of ZZ, $\mathrm{WZ}, \mathrm{WW}$, and $\mathrm{W} / \mathrm{Z}$ diboson production have been performed at a centre-of-mass energies of $7 \mathrm{TeV}$ and/or $8 \mathrm{TeV}$. No significan deviations from the SM have been observed. Unfolded differential cross sections are available for all fully leptonic results at $7 \mathrm{TeV}$. The measured cross sections are consistent with the SM predictions. The fiducia cross section measurements of electroweak $\mathrm{Z}+2 \mathrm{jets}$ production for proton-proton collision at $\sqrt{s}=8 \mathrm{TeV}$ using a dataset corresponding to an integrated luminosity of $20.3 \mathrm{fb}^{-1}$ collected by the ATLAS experiment at the LHC have been presented. The measured cross sections are in good agreement with the SM expectation. In addition, differential distributions and studies on hadronic activity have been shown. Also the cross section measurement in two fiducia regions with different sensitivity to the $\mathrm{EW}$ and the strong $\mathrm{WW}+2$ jets production in proton-proton collision at $\sqrt{s}=8 \mathrm{TeV}$, recorded by the ATLAS experiment has been shown. Also here a good agreement with the SM prediction is found.

\section{Acknowledgements}

The present work was co-funded by the European Union (European Social Fund ESF) and Greek national funds through the Operational Program "Education and Lifelong Learning" of the National Strategic Reference Framework (NSRF) 2007-1013. ARISTEIA-1893-ATLAS MICROMEGAS 


\section{References}

[1] ATLAS Collaboration, JINST, 3 S08003 (2008).

[2] L. Evans and P. Bryant (editors), JINST, 3 S08001 (2008).

[3] J. Campbell et al., [arXiv:1007.3492].

[4] P. M. Nadolsky et al., Phys.Rev. D78 (2008) 013004.

[5] M. Cacciari, G. P. Salam, G. Soyez, JHEP, 0804:063 (2008).

[6] ATLAS Collaboration, ATLAS-CONF-2012-124.

[7] ATLAS Collaboration, Phys.Rev. D87 (2013) 112003.

[8] T. Gleisberg et al. JHEP, 0902:007 (2009).

[9] M. L. Mangano, F. Piccinini, A. Polosa, M. Moretti, and R. Pittau, JHEP 0307 (2003) 11.

[10] ATLAS Collaboration, ATLAS-CONF-2014-033.

[11] Prerit Jaiswal, Takemichi Okui, [arXiv:1407.4537].

[12] Patrick Meade, Harikrishnan Ramani, Mao Zeng, [arXiv:1407.4481].

[13] ATLAS Collaboration, JHEP, 03 (2013) 128.

[14] ATLAS Collaboration, ATLAS-CONF-2012-090.

[15] ATLAS Collaboration, Eur. Phys. J. C72, 2173 (2012).

[16] ATLAS Collaboration, ATLAS-CONF-2013-021.

[17] ATLAS Collaboration, ATLAS-CONF-2012-157.

[18] ATLAS Collaboration, JHEP04(2014)031.

[19] ATLAS Collaboration, [arXiv:1405.6241].

[20] S. Alioli, P. Nason, C. Oleari and E. Re, JHEP 1006, 043 (2010).

[21] T. Melia, P. Nason, R. Rontsch and G. Zanderighi, Eur. Phys. J. C71, 1670 (2011), arXiv: 1102.4846[hep-ph].

[22] T. Sjostrand, S. Mrenna, and P. Skands, Comput. Phys. Comm. 178 (2008) 852.

[23] ATLAS Collaboration, ATL-PHYS-PUB-2012-003. 